\title{
Experiments on the Start and Stop of Windmill- and Cup-Anemometers with Particular Reference to Their Over-Estimation Factors
}

\author{
by \\ M. Sanuki \\ Meteorological Research Institute
}

(Received January 7, 1952)

\begin{abstract}
Three- and four-bladed windmill-anemometers and cupanemometers are started from rest in the wind tunnel and are also stopped from rotation in a still air, and their responses are compared with one another. The static torque coefficients are calculated from the observation of rotational speed versus time to be used in SCHRENK's parameter. SCHRENK's overestimation factors for both anemometers are evaluated into a single chart for rectangular wind waves. For sinusoidal wind waves, graphical solution of the equation of motion is carried out, from which the over-estimation factor is computed and compared with experiment due to DEACON. The phase lag is also obtained into a chart.
\end{abstract}

\section{Object of the present experiment and analysis}

After we have conducted a systematic investigation of the design of windmill anemometers [1], we have remarked a somewhat appreciable difference between the observed wind course with it and that with the ordinary cup-anemometer.

To clarify the cause, experiments on the stop of both types are carried out in the wind tunnel and in a still air. The anemometers are started from rest in the tunnel flow and also they are stopped from rotation in a still air. From these socalled dynamic experiments the static torque coefficients are calculated assuming some equations of motion. The analysis is further pushed on to calculate the overestimation factor. For this purpose the above-mentioned simple experiments are found to play an unexpectedly important role.

\section{The start of windmill-anemometer}

The equation of motion of a windmill-anemometer when started by a uniform wind speed can be written in the form 


$$
\left\{\begin{array}{l}
I \frac{d \omega}{d t}=c, \quad\left(0 \leq \omega \leq \omega_{\infty / 2}\right) \\
I \frac{d \omega}{d t}=c^{\prime}-c^{\prime \prime} \omega, \quad\left(\omega_{\infty / 2} \leq \omega \leq \omega_{\infty}\right)
\end{array}\right.
$$

where $I$ is the moment of inertia with respect to the axis of the anemometer, $\omega$ the angular velocity, $t$ time, $c, c^{\prime}$ and $c^{\prime \prime}$ are constants, and $\omega_{\infty}$ is the final angular velocity corresponding to the wind speed $V$. It is seen the torque of a windmill changes against the rotation ratio $\omega R / V$ ( $R$ is the windmill radius) for blade angles near $60^{\circ}$ at $75 \%$ radius as shown in Fig. 1, (half-stalled condition, Reference 1), and the constants $c, c^{\prime}$ and $c^{\prime \prime}$ may be written as

$$
\left\{\begin{array}{l}
c=c_{d_{0}} \frac{1}{2} \rho V^{2} \cdot \pi R^{2} \cdot R, \\
c^{\prime}=2 c, \\
c^{\prime \prime}=2 c / \omega,
\end{array}\right.
$$

where $c_{d_{0}}$ is the dimensionless torque coefficient, $\rho$ the air density and $V$ the wind speed.

Equation (1) can easily be integrated and we get

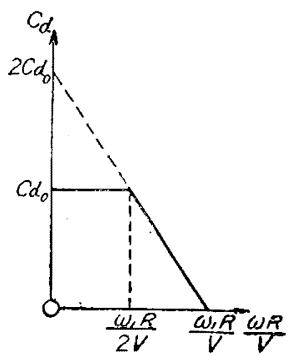

Fig. 1. Torque coefficient of half-stalled windmill $\left(\omega_{1} \equiv \omega_{\infty}\right)$.

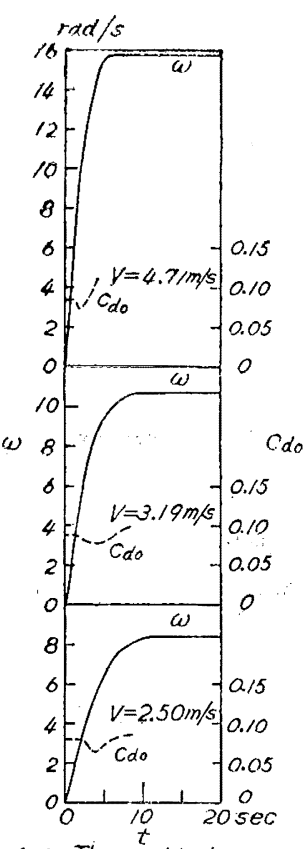

(a) Three-blader.

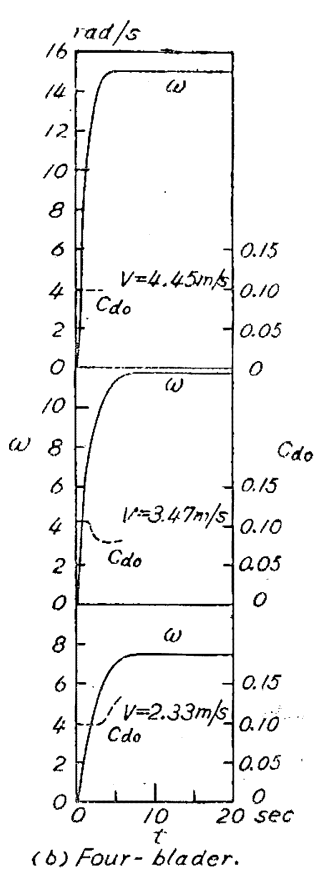

(b) Four-blader.

$$
\left\{\begin{array}{r}
\omega=\frac{c}{I} t, \quad\left(0 \leq \omega \leq \omega_{\infty / 2}\right), \\
\omega==\frac{1}{c^{\prime \prime}}\left\{c^{\prime}+\left(c^{\prime \prime} \omega_{0}-c^{\prime}\right) e^{-\frac{c^{\prime \prime}}{I}}\right\}, \\
\quad\left(\omega_{\infty / 2} \leq \omega \leq \omega_{\infty}\right),
\end{array}\right.
$$

where $\omega_{0}$ is the value of $\omega$ at $t=0$. From the second solution of (3) we see $\omega=c^{\prime} / c^{\prime \prime}=\omega_{\infty}$ at $t=\infty$.

If a windmill is started from rest in a wind of uniform speed of $V$, and $\omega$ is observed at each time interval, the constants $c$ and $c^{\prime}$ (also $c^{\prime \prime}$ ) can be calculated and the starting torque coefficient $c_{\pi_{0}}$ may be obtained.

In Fig. 2 are shown the experiments conducted for Speedovane with threeand four-blader windmills (their forms and dimensions are given in Reference [2]) in the 1.5-meter wind tunnel of the Meteorological Research Institute. It is observed that the four-blader is superior to the three-blader in start, and the final indication is obtained in $70 \%$ 
of time of that for the latter. The calculated starting torque coefficient $c_{\alpha_{0}}$ is correspondingly, about 15\%, larger for the four-blader than the three-blader.

The starting-torque coefficient $c_{0}$ is, on the other hand, tested in the wind tunnel as shown in Fig. 3. In this test the three-blader gives larger values of $c_{l_{0}}$ than the four-blader by about $15-20 \%$. This fact is somewhat questionable and the reason is not yet well explored. 'The moment of inertia $I$ is 0.000324 $\mathrm{kgms}^{2}$ for the three-blader and $0.000273 \mathrm{kgms}^{2}$ for the four-blader, but they do not come into the calculation of $c_{d_{0}}$. The developed surface area $A$ is the same for both windmill blades, given by

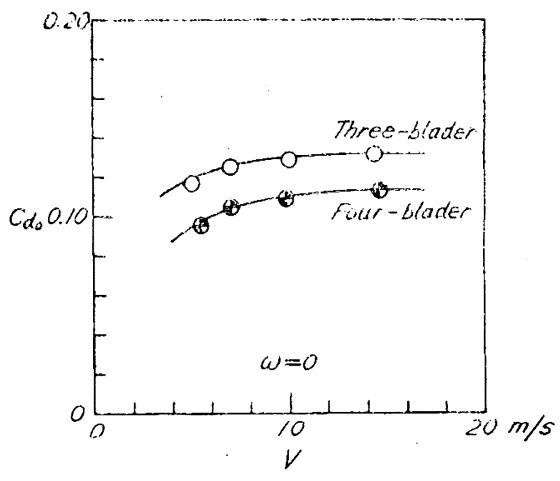

Fig. 3. Static torque coefficient of Speedovane. (Windmill diameter $350 \mathrm{~mm}$, M. R. I wind tunnel.)

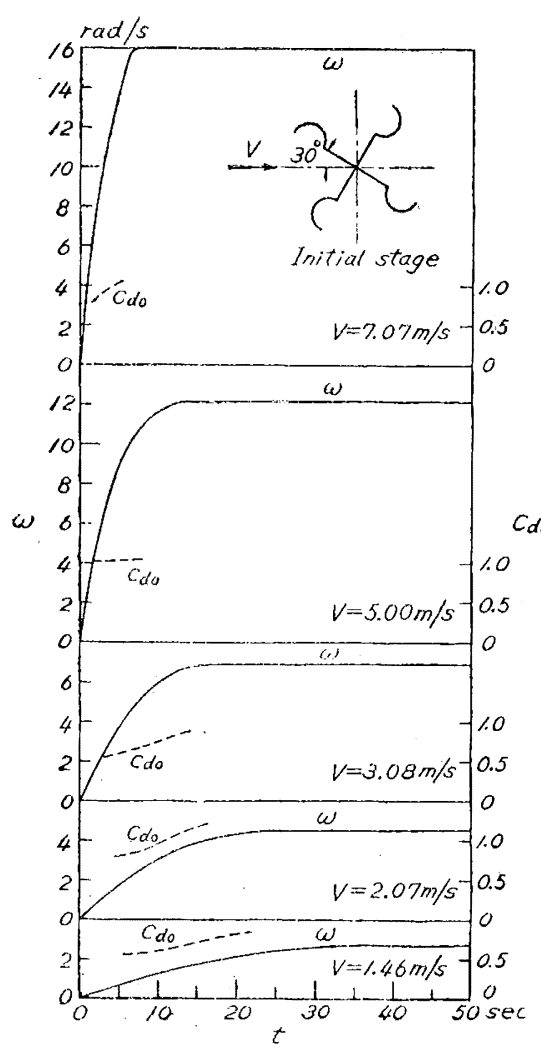

Fig. 4. Start of cup-anemometer in various wind speeds, and calculated starting torque coefficient (M. R.I. wind tunnel).

$$
A=p \int_{-\frac{\pi}{p}}^{\frac{\pi}{p}} \frac{1}{2} r^{2} d \theta=R^{2},
$$

as the plan-form of the windmill blades is designed after the following formula in polar coordinates $(r, \theta)$

$$
r^{2}=R^{2} \cos p \theta,
$$

where $p$ is the number of blades and $R=0.175 \mathrm{~m}$ in the present case.

\section{The start of cup-anemometer}

The equation of motion of a cup-anemometer in start by a uniform wind speed $V$ is written [3] as

$$
I_{--}^{d \omega}=c^{\prime}-c^{\prime \prime} \omega
$$

where $c^{\prime}$ and $c^{\prime \prime}$ are constants. The solution has the same form as the second type of Equation (3). The constants can now be written in the form

$$
\left\{\begin{array}{l}
c^{\prime}=c_{a_{0}} \cdot \frac{1}{2} \rho V^{2} \cdot p \pi R_{0}^{2} \cdot R, \\
c^{\prime \prime}=c^{\prime} / \omega_{\infty},
\end{array}\right.
$$

where $p$ is the number of cups, $c_{t_{0}}$ the dimensionless starting torque coefficient, $R_{0}$ the cup radius, $R$ the cup center radius and other 
notations are the same as before. In the present case, $I=0.00139 \mathrm{kgms}^{2}, p=4$, $R_{0}=0.04 \mathrm{~m}, R=0.14 \mathrm{~m}$. 'The wind tunnel test results are illustrated in Fig. 4 . The start is found to be most favourable when the cup arm is inclined at $45^{\circ}$ to the wind direction in its initial stage, but the time necessary to attain the final rotating speed does not differ much for the initial cup positions. In the figure an example of $30^{\circ}$ inclination is shown.

It is seen that the cup-anemometer requires about three times as much time as Speedovane with four-bladed windmill to obtain the same final rotating speed. The values of $c_{i_{0}}$ increase with increasing $\omega$ showing the effect of bearing friction. Moreover, $c_{\dot{d}_{0}}$ increases with $V$, which fact can be explained by the change of flow pattern with Reynolds number. American wind tunnel test on four-cup wheel [4] gives $0.59 \sim 0.56$ for $V=9 \sim 15 \mathrm{~m} / \mathrm{s}, R_{0}=0.508 \mathrm{~m}, R=0.236 \mathrm{~m}$, which values are in good conformity to our results for lower $V$.

\section{The stop of windmill-anemometer}

The equation of motion of a rotating windmill-anemometer in a still air may be written in the form

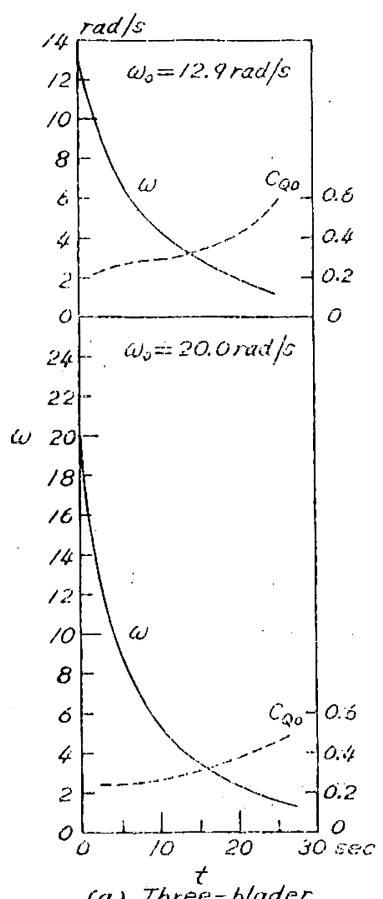

(a) Three-blader

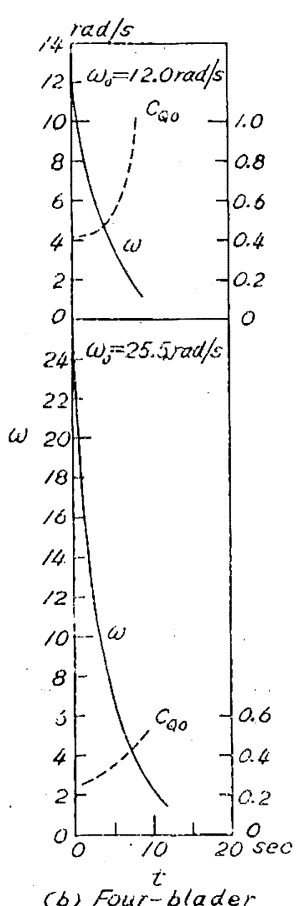

(b) Four-blader

Fig. 5. Stop of Speedovane with various initial angular velocities in a still air.

$$
I \frac{d \omega}{d t}=-c \omega^{2}
$$

where $c$ is a constant and other notations are the same as before. $c$ can be written as

(9) $c=C_{Q_{0}} \rho n^{2} D^{5} / \omega_{2}=\frac{8}{\pi^{2}}-C_{Q_{0}} \rho R^{5}$,

where $C_{Q_{0}}$ is the dimensionless static torque coefficient used in the experimental expression of propellers, $n$ the number of revolution, and $D$ the windmill diameter.

In a still air the Speedovane is brought to rest from an initial rotation, and the angular velocity $\omega$ is observed for each time interval as illustrated in Fig. 5, where $\omega=1 \mathrm{rad} / \mathrm{s}$ corresponds to about $V=0.30 \mathrm{~m} / \mathrm{s}$. It is seen that the three-blader needs $100 \sim 200 \%$ more time than the fourblader before it stops. From the observations the values of $c$ and 
therefore $C_{Q_{0}}$ are calculated from the solution of Equation (8), viz.,

$$
\omega=\frac{I \omega_{0}}{c \omega_{0} t+I}
$$

where $\omega_{0}$ is the initial angular velocity. The values of $C_{Q_{0}}$ rapidly increase with decreasing $\omega$ indicating the bearing friction effect.

The order of $C_{Q_{0}}$ thus obtained can be compared with the experimental results for propellers. 'The three-bladed propeller 5868-9 [5] gives $C_{Q_{0}}=0.087$ for blade angle $60^{\circ}$ (i.e., the blade angle of the Speedovane blades) at $75 \%$ radius. If the torque is assumed to be proportional to the blade width we get $C_{Q_{0}}=0.27$ for the Speedovane threeblader which value is in good coincidence with the present experimental result for higher rotational speed, as seen in Fig. 5 a.

\section{The stop of cup-anemometer}

The equation of motion of a rotating cup-anemometer in a still air is written in the form

$$
I \frac{d \omega}{d t}=-c \omega^{2}
$$

where $c$ is a constant and other notations are the same as before. $c$ can be expressed as

$$
c=C_{D} \cdot \frac{1}{2} \rho V^{2} p \pi R_{0}^{2} \cdot R,
$$

where $p$ is the number of cups, $C_{D}$ the dimensionless drag coefficient of the cup, $R_{0}$ the cup radius, and $R$ the cup center radius.

The cup-anemometer is brought to rest in a still air from an initial rotational speed $\omega_{0}(\omega=1 \mathrm{rad} / \mathrm{s}$ corresponds to $V=\mathrm{ca} .0 .37 \mathrm{~m} / \mathrm{s})$, and from Equation (11) the values of $c$ or $C_{D}$ are calculated as illustrated in Fig. 6. The cup-anemometer requires a remarkably longer time than the Speedovane before it is brought to rest.

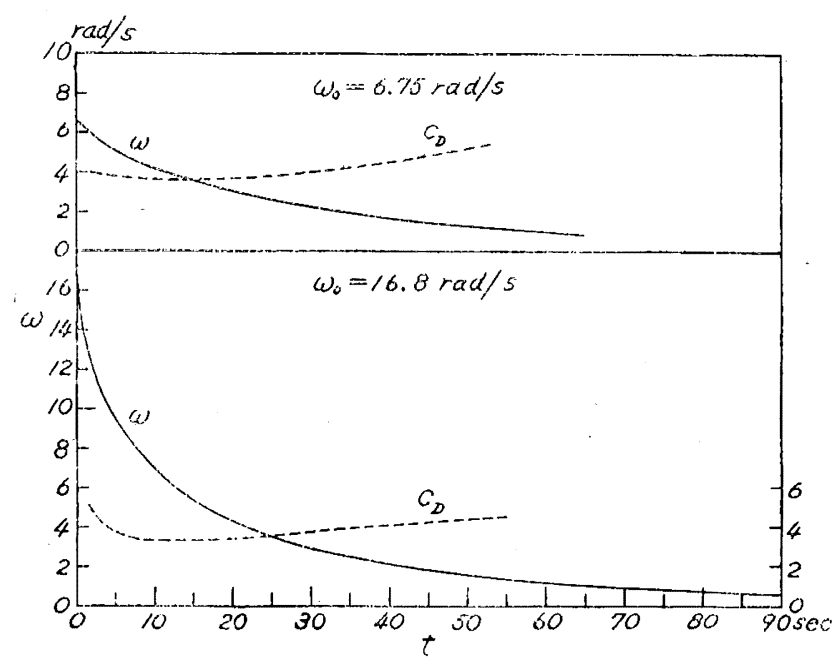

Fig. 6. Stop of cup-anemometer with various initial angular velocities in a still air.

The order of $C_{D}$ thus obtained may be checked by experimental results. An American experiment [4] shows that the drag coefficient of a hemispherical cup as $C_{D}=1.4$. The present experiment gives about $C_{D}=3.5$. The difference might be 
attributed to the acrodynamic interference or other causes.

The cup type requires much more time than the windmill type before it stops. For each type the time taken before it stops is nearly constant in spite of the initial angular velocity differences.

\section{Frictional effect}

The bearing friction is included in the constants in the equations of motion. The static friction torque is of the order of $10 \mathrm{grcm}$ for the windmill type and $2 \mathrm{grcm}$ for the cup type. Therefore the effect is never negligible except for higher values of $d \omega / d t$ and the obtained values of constants comprise both aerodynamic and mechanical effects.

The friction decreases as angular velocity increases. If we consider the static friction torque as the upper limit of mechanical effect, it influences the total accelerating or decelerating torque by more than $10 \%$ for the range $d \omega / d t \leq 0.84 / \mathrm{s}^{2}$ for the three-blader windmill type, $d \omega / d t \leq 1.09 / \mathrm{s}^{2}$ for the four-blader, and $d \omega / d t \leq 0.14 / \mathrm{s}^{2}$ for the cup type. And the mechanical effect plays the chief role for the range $d \omega / d t \leq 0.084 / \mathrm{s}^{2}$ for the three-blader, $d \omega / d t \leq 0.109 / \mathrm{s}^{2}$ for the four-blader, and $d \omega / d t \leq 0.014 / \mathrm{s}^{2}$ for the cup type. Naturally these limits seem to be too severe for actual cases, but as no reasonable relations are yet found between frictional torque and angular velocity, the above values represent only cautious margins.

\section{Over-estimation of anemometer indication}

Next we shall consider the over-estimation of anemometer indication in general. We assume the wind speed fluctuation as shown in Fig. 7 (rectangular wind waves), where $V_{0}$ is the mean wind speed, $\Delta V$ the amplitude and $T$ the period.

The accelerating torque coefficient of anemometer is also assumed as

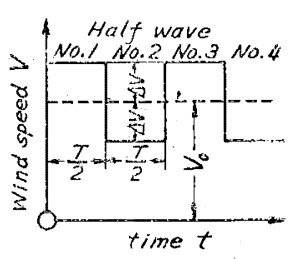

Fig. 7. Wind fluctuation.

$$
c_{a l}=\text { torque } / \frac{1}{2} \rho V^{2} \cdot \pi K^{2} \cdot R=2 c_{t_{0}}-2 c_{i_{0}} \lambda_{0} \frac{\omega R}{V^{-}},
$$

for the windmill type, neglecting the friction. $c_{l}$ is the static torque coefficient for the speed ratio $V / \omega R=\infty$, and the speed ratio in equilibrium $\lambda_{0}=V_{0} / \omega_{0} R, \omega_{0}$ being the rotational speed corresponding to $V_{0}$.

For the cup type we take

$$
c_{d}=\text { torque } / \frac{1}{2} \rho V^{2} \cdot p \pi R_{0}^{2} \cdot R=c_{i_{0}}-c_{i_{0}} \lambda_{0} \frac{\omega R}{V},
$$

where $c_{a_{0}}$ is the static torque coefficient.

The braking torque coefficient is usually written for the windmill type in the form 


$$
k_{d}=\text { torque } / \frac{1}{2} \rho \omega^{2} R^{2} \cdot \pi R^{2} \cdot R=c_{d}\left(\frac{V}{\omega R}\right)^{2} .
$$

The same relation holds also for the cup type if we define $k_{d}=$ torque $/ \frac{1}{2} \rho \omega^{2} R^{2} \cdot p \pi R_{0}^{2} \cdot R$. By this transformation Equation (13) becomes

$$
k_{d}=2 c_{\pi 0}\left(\frac{V}{\omega R}\right)^{2}-2 c_{a_{0}} \lambda_{0} \frac{V}{\omega R},
$$

for the windmill type if we wish continuous variation of torque coefficient near equilibrium state at $c_{t}=0$ and $V / \omega R=V_{0} / \omega_{0} R=\lambda_{0}$. For the cup type we get

$$
k_{a}=c_{d_{0}}\left(\frac{V}{\omega R}\right)^{2}-c_{a_{0}} \lambda_{0} \frac{V}{\omega R} .
$$

Fig. 8 illustrates $c_{d}$ versus $\omega R / V$ and $k_{d}$ versus $V / \omega R$ together with the experimental results on cup-anemometers due to SCHRENK [7].
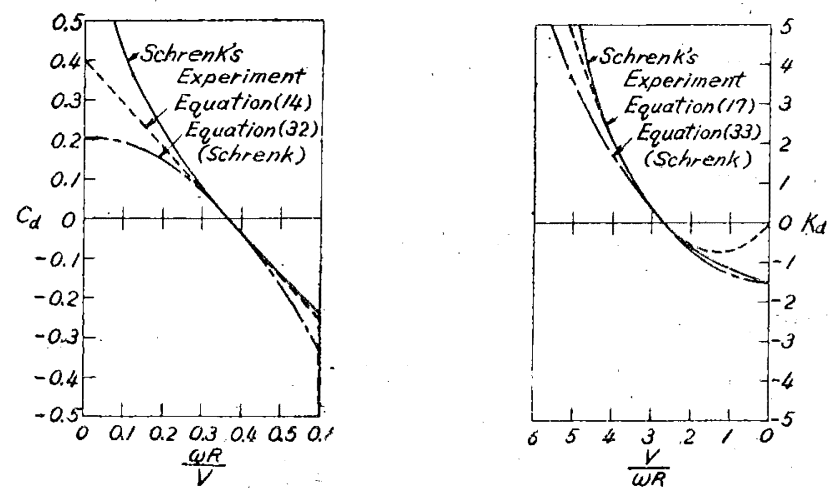

Fig. 8. Approximate expressions of cup-anemometer torque coefficients.

The rotational speed $\omega_{2 n-1}$ corresponding to the odd $(2 n-1)$-th half wind wave end can be written from Equation (3) after some calculations as

$$
\frac{\omega_{2 n-1}}{\omega_{0}}=1+S+2 S e^{-2 K \pi}\left\{\frac{1-e^{-2(n-1) K \pi}}{1-e^{-2 K \pi}}\right\}\left\{1-e^{K \pi(1-S)}\right\}-S e^{-K \pi(2 n-1+S)},
$$

where $S=\Delta V / V_{0}$, and $n=1,2,3, \cdots \cdots$.

The rotational speed $\omega_{3 n}$ corresponding to the even $2 n$-th $(n=1,2,3, \cdots \cdots)$ half wind wave end is obtained in the similar manner as

$$
\frac{\omega_{3 n}}{\omega_{0}}=1-S-2 S e^{-2 K \pi}\left\{\frac{1-e^{-2 n K \pi}}{1-e^{-2 K \pi}}\right\}\left\{1-e^{K \pi(1+S)}\right\}+S e^{-2 n K \pi} .
$$

The over-estimation factor corresponding to the odd $(2 n-1)$-th half wind wave (N.B. not the accumulative over-estimation but a single one for the half wave concerned) is defined and calculated as

$$
\begin{aligned}
Z_{t, 2 n-1} & =\left\{\frac{2}{T} \int_{0}^{T / 2} \omega d t-\omega_{0}\right\} / \omega_{0} \\
= & S+\frac{1}{K \pi(1+S)}\left\{\frac{\omega_{2 n-2}}{\omega_{0}}-(1+S)\right\}\left\{1-e^{-K \pi(1+S)}\right\},
\end{aligned}
$$


where $t$ denotes time, and $K$ is SCHRENK's parameter defined by

$$
K=2 c_{d_{0}} \lambda_{0} \rho V_{0} T R^{4} / 4 I,
$$

for the windmill type and

$$
K=p c_{a_{0}} \dot{\lambda}_{0} \rho V_{0} T R_{0}{ }^{2} R^{2} / 4 I
$$

for the cup type. It is to be noted here that $p c_{t_{0}}$ and $\lambda_{0}$ are used in the same meaning as SCHRENK's aerodynamic coefficients $\alpha$ and $\sqrt{\beta / \alpha}$.

Similarly for the even $2 n$-th half wind wave we have

$$
Z_{t, 2 n}=-S+\frac{1}{K \pi(1-S)}\left\{\frac{\omega_{2 n-1}}{\omega_{0}}-(1-S)\right\}\left\{1-e^{-K \pi(1-S)}\right\} .
$$

The over-estimation factor for the $n$-th complete wave should be taken as $\left(Z_{t, 2 n-1}+Z_{t, 2 n}\right) / 2$.

If $n$ is taken to be infinity the rotational speed tends to

$$
\operatorname{Lim}_{n=\infty} \frac{\omega_{2} n-i}{\omega_{0}}=1+S+2 S e^{-2 K \pi}\left\{\frac{1-e^{K \pi(1-S)}}{1-e^{-2 K \pi}}\right\},
$$

for the odd half wind wave end and to

$$
\operatorname{Lim}_{n=\infty} \frac{\omega_{n n}}{\omega_{0}}=1-S-2 S e^{-2 K \pi}\left\{\frac{1-e^{K \pi(1+S)}}{1-e^{-2 K_{n}}}\right\},
$$

for the even half wind wave.

The over-estimation factor becomes in each case

$$
\operatorname{Lim}_{n=\infty} Z_{t, n-1}=S-\frac{2 S}{\bar{K} \pi(1+S)\left(1-e^{-2 K \pi}\right)}\left\{1-e^{-K \pi(1+S)}\right\}\left\{1-e^{-K \pi(1-S)}\right\},
$$

for the odd half wind wave, and

$$
\operatorname{Lim}_{n=\infty} Z_{t, 2 n}=-S+\frac{2 S}{K \pi(1-S)\left(1-e^{-2 \bar{K} \pi}\right.}\left\{1-e^{-K \pi(1+S)}\right\}\left\{1-e^{-K \pi(1-S)}\right\},
$$

for the even half wind wave.

The over-estimation factor for the one complete wind wave after infinite series of waves is finally given by

$$
Z_{t}=\operatorname{Lim}_{n=\infty} \frac{Z_{t_{*}, n-1}+Z_{t, n}}{2}=\frac{2 S^{2}\left\{1-e^{-K \pi(1+S)}\right\}\left\{1-e^{-K \pi(1-S)}\right\}}{K \pi\left(1-S^{2}\right)\left(1-e^{-2 K \pi}\right)} .
$$

The value of $Z_{t}$ thus defined has the same meaning as that adopted by SCHRENK. As limiting cases we have

$$
\operatorname{Lim}_{K=0} Z_{t}=S^{2}
$$

and

$$
\operatorname{Lim}_{K=\infty} Z_{t}=0 .
$$

In Fig. 9 the over-estimation factor $Z_{t}$ is illustrated against $K$. Comparing this chart with that given by SCHRENK we immediately notice that our calculated values of $Z_{t}$ are markedly larger that those of SCHRENK. This discrepancy arises 


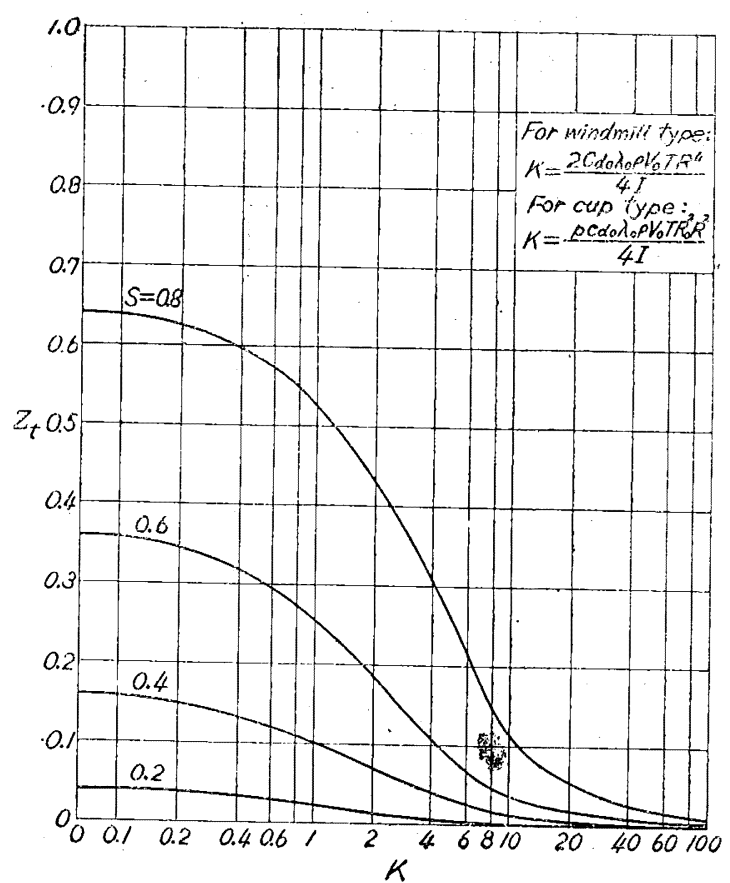

Fig. 9. Over-estimation factor for rectangular wind waves.

or:

$$
c_{\bar{a}}=c_{a_{0}}-c_{i_{0}} \lambda_{v}{ }^{2}\left(\frac{\omega R}{V}\right)^{2}
$$

$$
k_{t l}=c_{i_{0}}\left(\frac{\mathrm{V}}{\omega R}\right)^{2}-c_{i_{0}} \lambda_{0}{ }^{2},
$$

we get, in case of $K=0$ and rectangular wave,

$$
Z_{t}=\sqrt{1+S^{2}}-1 \text {. }
$$

The over-estimation factors for $K=0$ obtained form Equations (29), (31) and (34) together with SCHRENK's calculation for sinusoidal wind fluctuation

$$
Z_{t}=\sqrt{1+S^{2} / 2}-1
$$

are illustrated in Fig. 10.

The value of $Z_{t}$ differs much acccording to the form of anemometer torque coefficient formula assumed, and also according to the mode of wind fluctuation, but the reasonable margin of $Z_{t}$ seems to exist a little higher (SCHRENK employed a factor 1.4) than the lowest curve in Fig. 10. If, therefore, we modify the curves ix: Fig. 9 by multiplying with some factor according to Fig. 10, we

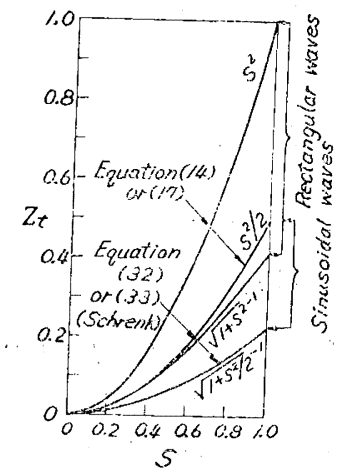

Fig. 10. Over-estimation factors for $K=0$.

* S corresponds to $S$ of Schrenk. 
shall be able to obtain a chart quite similar to that given by SCHRENK.

Before concluding this section, one more fact should be pointed out. The foregoing over-estimation factor is concerned with the terminal state after infinite

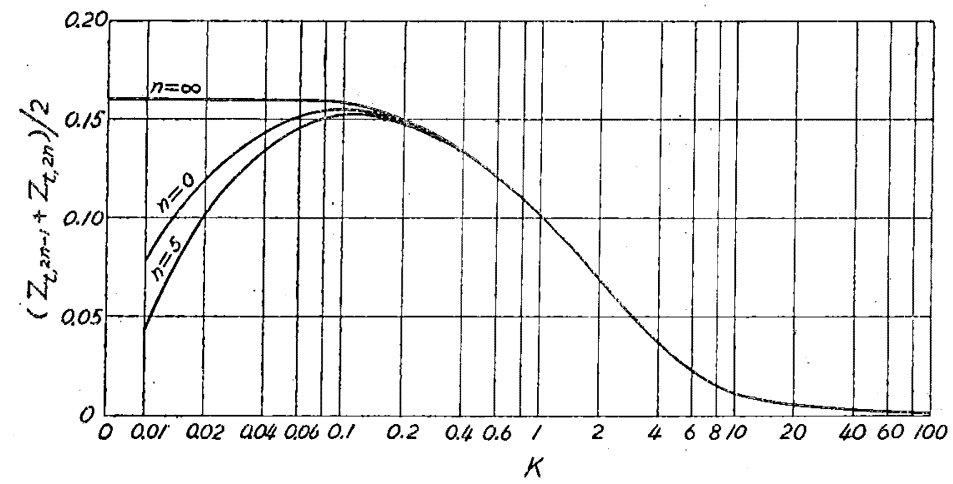

Fig. 11. Over-estimation factor for the state after ninite number $n$ of wind waves. (Rectangular wind waves, cf. Fig. 9).

series of wind waves. But Equations (20) and (23) stand for cases of a finite number of waves. The over-estimation factor $\left(Z_{t, 2 n-1}+Z_{t, 2 n}\right) / 2$ is calculated and illustrated in Fig. 11 for $n=5,10$ and $\infty$ for rectangular wind waves without any correction. The effect of the number of waves is marked only for lower region of $K$ which will be realized by the anemometer in case of slow wind speed with high frequency.

For the same reason the over-estimation is more pronounced for lower air density. Mountain stations will suffer more from the overrunning of the anemometer than stations situated on the plain.

\section{Graphical solution of the equation of motion}

SCHRENK has solved by an approximate method the equation of motion of a cup type

$$
I \frac{d \omega}{d t}=\frac{1}{2} c_{a l} V^{2} \cdot p_{\pi} R_{0}{ }^{2} \cdot R,
$$

where $c_{d}$ is given in Equation (32). This equation can be written as

$$
\frac{d \omega_{1}}{d t_{1}}=2 \pi K\left\{\left(1+S \sin 2 \pi t_{1}\right)^{2}-\omega_{1}^{2}\right\},
$$

where $\omega_{1}=\omega / \omega_{0}, t_{1}=t / T$, and the sinusoidal variation of $V$ is assumed as before. Equation (37) is non-linear and the present author solved it by the isocline rethod described in Reference [8]. An example of the graphical solution for $K=1, S=0.5$ is shown in Fig. 12. The variation of the anemometer speed $\omega_{1}=$ $\omega / \omega_{0}$ against the time $t_{1}=t / \%$ is almost sinusoidal with a certain phase lag from the wind wave. 


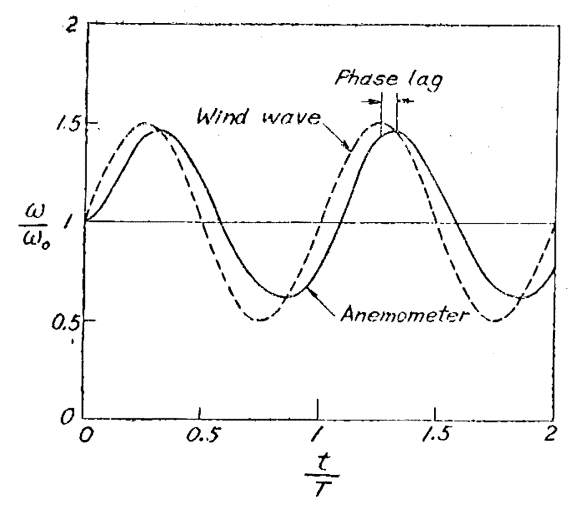

Fig. 12. Computed rotational speed of anemometer for sinusoidal wind waves ( $K=1, S=0.5)$.

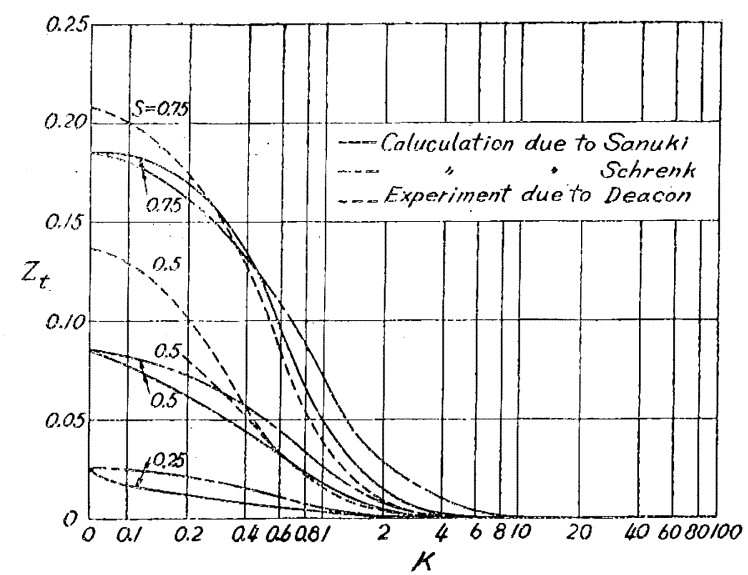

Fig. 13. Computed over-estimation factor for sinusoidal wind waves compared with the experiment due to DEACON.

The over-estimation factor $Z_{t}$ defined in Equation (28) after a certain number of waves is computed in Fig. 13, together with that calculated by SCHRENK, and experiment by DEACON [9]. The calculation by the present author is multiplied with the factor 1.4 for the same reason as given by SCHRENK. Better coincidence with the experiment for quasi-sinusoidal wind waves is found than the calculation due to SCHRENK, which is somewhat arbitrary interpolation between two limiting cases of $K$. According to this result, SCHRENK seems to have employed the factor 1.4 with fairly acceptable reason except the intermediate ambiguity in his calculation.

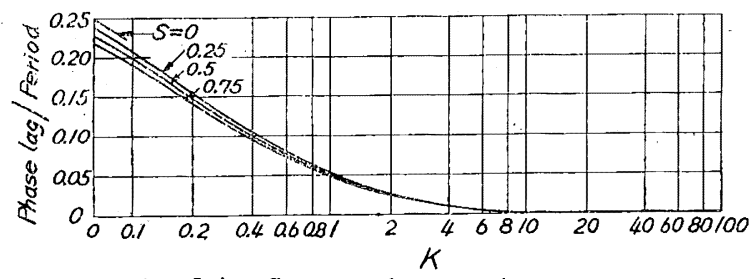

Fig. 14. Computed phase lag chart.

Further the phase lag defined in Fig. 12 is computed as illustrated in Fig. 14. The limiting case for $S=0$ is also added, for which a quarter-period phase lag is to be expected for $K=0$.

On this occasion it is to be stressed that the present discussion applies equally to the windmill-anemometer if we write

$$
K=c_{\pi_{0}} \lambda_{0} \rho V_{0} T R^{4} / 4 I
$$

instead of Equation (21), based on the assumption of parabolic variation of torque coefficient.

In both cases of cup and windmill types the experimental determination of $c_{d_{0}}$ described in Articles 2 to 5 affords simple and reasonable basis to the calculation of SCHRENK's parameter $K$. The evaluation of $c_{\pi_{0}}$ was based on linear variation of 
torque coefficient but may safely be applied also to SCHRENK's cases.*

On the whole, the over-estimation of the windmill-anemometer seems to be far less than that of cup type, as the value of $K$ for the former ranges from 0.020 to 200 for $V_{0}=1 \mathrm{~m} / \mathrm{s}, T=1 \mathrm{~s}$ and $V_{0}=100 \mathrm{~m} / \mathrm{s}, T=100 \mathrm{~s}\left(c_{d_{0}}=0.10, \lambda_{0}=1.69, \rho=1 / 8 \mathrm{~kg} \mathrm{~m}^{-4} \mathrm{~s}^{2}\right.$, $R=0.175 \mathrm{~m}, I=0.000273 \mathrm{~kg} \mathrm{\textrm {ms } ^ { 2 }}$ for four-blader $)$, whereas for the latter $K=0.0030$ to 30 also for the same legends $\left(c_{d_{0}}=0.40, \lambda_{0}=2.65, \mathrm{R}_{0}=0.04 \mathrm{~m}, R=0.14 \mathrm{~m}, I=0.00139 \mathrm{~kg} \mathrm{~m} \mathrm{~s} \mathrm{~s}^{2}\right.$, $p=4$ ) respectively. And further, if the windmill type should follow the wind with less than $5 \%$ over-estimation and also $5 \%$ phase lag even in the wind waves of $V_{0}=1 \mathrm{~m} / \mathrm{s}, T=1 \mathrm{~s}$, the moment of inertia must be reduced to $1 / 50$ of the present value (cf. Figs. 13 and 14).

\section{Conclusions}

From the experimental results are deduced the following conclusions:

1) The overrunning is more pronounced than the underrunning both for windmill- and cup-anemometer.

2) The windmill type is superior to the cup type with respect to the response.

3) The four-blader windmill is superior to the three-blader in the same sense. From the theoretical calculations are to be shown:

4) Linear expression of the anemometer accelerating torque coefficient gives, for square wind waves, much larger over-estimation factor than that given by SCHRENK for sinusoidal wind waves.

5) The effect of finite number of wind waves is only conspicuous for smaller values of SCHRENK.'s parameter.

6) Graphical solution of the equation of motion gives somewhat different values of over-estimation factor from those given by the approximate method due to SCHRENK. As regards the agreement with the experiment by DEACON, the former values are better.

7) The phase lag is larger for smaller amplitudes of the wind fluctuations.

8) The start and stop method affords simple and reasonable determination of aerodynamic factors in SCHRENK's parameter.

Acknowledgment - - The present experiment was carried out by Mr. S. KIMURA and part of the theoretical evaluation was contributed by Mr. K. NAITO, both members of our Laboratory.

* Of course, the direct integration of Equation (37) is possible in case of start and stop, which gives more reasonable value of $K$. 


\section{References}

[1] Sanuki, M. 1950: Studies on Biplane Wind Vanes, Ventilator Tubes and Cup-Anemometers (II), Papers in Meteorology and Geophysics, 1, p, 282, Figs. 50-51.

[2] Sanuki, M., Krmura, S. and Tsuda, N. 1951: Studies on Biplane Wind Vanes, Ventilator Tubes and Cup-Anemometers (Supplementary Report), Papers in Meteorology and Geophysics, 2, p. 317

[3] Sanukr, M., 1950: Studies on Biplane Wind. Vanes, Ventilater Tubes and Cup-Anemometers (I) Papers in Meteorology and Geophysics, 1 p. 81, Equation (8)

[4] BrevoorT, M. J. and Joyner, U. T. 1935; Experimental Investigation of the RobinsonType Cup-Anemometer, N. A.C. A. Technical Report 513, Fig. 21.

[5] Bimmann. D. and Haktman, E. P. 1939: N. A. C. A. Technical Report 658.

[6] Brevookt and Joyner: loc. cit., Fig. 4.

[7] Schrenk, O. 1929: Ueber die Traegheitsfehler des Schalenkreuz-Anemometers bei schwankender Windstaerke, Zeitschrift für technisch Physik 10.

[8] Mcrachlan, N. W. 1950: Ordinary Non-Linear Differential Equations in Engineering and Physical Sciences (The Clarendon Press, Oxford).

[9] Dracon, E. L. 1951: The Over-Estimation Error of Cup Anemomgters in Fluctuating Winds, Journal of Scientific Instruments 28. 